\title{
Replacement of the aortic root: Does only size matter?
}

\author{
Jean E. Bachet, MD, FEBCTS
}

\author{
$\overline{\text { From ADETEC}}$, Suresnes, France. \\ Disclosures: Author has nothing to disclose with regard to commercial support. \\ Received for publication July 29, 2018; accepted for publication July 30, 2018; available ahead of print Sept 6 , \\ 2018. \\ Address for reprints: Jean E. Bachet, MD, FEBCTS, ADETEC, 1 Place Marcel Legras, 92150 Suresnes, France \\ (E-mail: jean.bachet@yahoo.fr). \\ J Thorac Cardiovasc Surg 2019;157:487 \\ $0022-5223 / \$ 36.00$ \\ Copyright (C) 2018 by The American Association for Thoracic Surgery \\ https://doi.org/10.1016/j.jtcvs.2018.07.088
}

Ploneck and colleagues ${ }^{1}$ analyzed the difficulty of properly measuring the size of an aortic root and propose several modes of assessing the actual dimensions of this anatomic feature.

The methodology proposed by the authors seems simple and appropriate. In particular, the proposition of assessing the actual dimensions of a root and of each sinus of Valsalva through "cross-sections of the corresponding aortic segments on planes perpendicular to the long axis of the vessel" ${ }^{1}$ appears to be common sense and quite realistic. So is their proposition to systematically use 3-dimensional multiplanar reconstructions of computed tomography angiography images in preference to transthoracic echocardiography or standard bidimensional computed tomography scans.

The authors conclude: "Therefore, we suggest that a standardized method presented in this article should be used to assess the aortic root and avoid underestimation of the maximum aortic root dimension-a potentially dangerous diagnostic mistake."

As a whole, it appears that the difference in measurements observed and reported by the authors are somewhat reduced (about 5-7 $\mathrm{mm}$ in diameter and $7 \mathrm{~cm}^{2}$ in the root surface). A difference of $20 \mathrm{~mm}$ was found in only 1 patient out of $112(<1 \%)$. This is too small a proportion to be significant.

One may wonder whether those reduced differences could really interfere in the indication of surgery. Indeed, clinical practice and analysis of the literature tend to show that in the majority of patients (except in those with connective tissue disease), surgery on the aortic root is indicated in front of another pathology-in particular severe aortic valve regurgitation or stenosis. In those cases, the presence of an obviously dilated root on the transthoracic or 2-dimensional computed tomography scan images will certainly indicate replacement. In case of uncertainty, the method proposed by Ploneck and colleagues is generally carried out and certainly helps in solving the problem. But, in most cases, if there is a surgical indication because of the associated pathology, a surgeon can intraoperatively

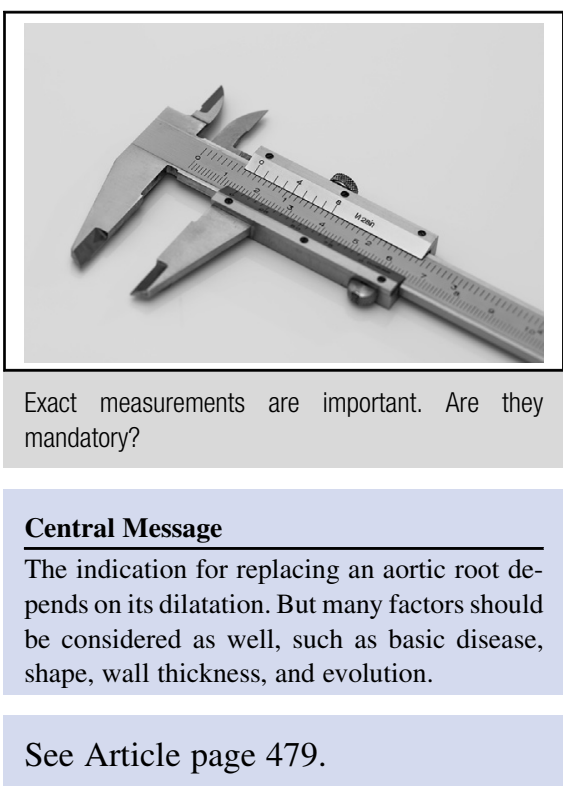

analyze the shape and the quality of the aortic root, exactly measure its dimensions, assess the thickness and quality of the aortic wall and the shape and dimensions of the sinuses, and ultimately decide whether or not a root replacement is indicated. Finally, in cases where there is no pressing surgical indication, the evolution of the root dimensions at repeated assessments on an every 6 months or yearly basis, for instance, generally helps in making a decision.

If, on the other hand, we consider the risk of sudden severe accident and, in particular, the occurrence of acute type A dissection, we may notice that in a large enough majority of patients the aortic root and the ascending aorta are not dilated and remain within normal anatomic dimensions. Except in patients with a connective tissue disease or a family history of acute dissection or sudden cardiovascular death, the risk is mostly linked to the presence of chronic or acute arterial hypertension due to exceptional events. In those patients, the aortic root dimensions are never measured before the accident and a difference of a few millimeters does not change the obvious and mandatory surgical indication.

Will a more sophisticated method of measuring root dimensions significantly reduce the rate of severe accidents? The question is still open.

\section{Reference}

1. Plonek T, Berezowski M, Bochenek M, Filip G, Rylski B, Golesworthy T, Jasinski M. A comparison of aortic root measurements by echocardiography and computed tomography. J Thorac Cardiovasc Surg. 2019;157:479-86. 